\title{
Heart Rate Changes in Pet and Lab Dogs as Response to Human Facial Expressions
}

\author{
Anjuli L. A. Barber*, Ester M. Müller, Dania Randi, Corsin A. Müller, Ludwig Huber \\ Comparative Cognition, Messerli Research Institute, University of Veterinary Medicine Vienna, Medical \\ University Vienna, University of Vienna, Vienna, Austria \\ *Corresponding Author: Anjuli L. A. Barber, Comparative Cognition, Messerli Research Institute, \\ University of Veterinary Medicine Vienna, Medical University Vienna, University of Vienna, Vienna, \\ Austria, Email: anjuli.barber@vetmeduni.ac.at
}

\begin{abstract}
Dogs are an outstanding model to study heterospecific communication. They react contagiously to human visual and acoustic stimuli and can discriminate between facial expressions of humans. However, their physiological reactions to emotional relevant stimuli are still not well understood. This study examined the cardiac responses of domestic dogs upon seeing faces of humans that differed with respect to emotional expression (angry, happy, sad, neutral). Because the response to human emotions is likely influenced by individual experience with human faces, we compared the cardiac responses of dogs with plenty of experience with humans (pet dogs) and dogs with little experience with humans (lab dogs) upon seeing familiar and unfamiliar faces. We found significant differences between the pet and the lab dogs concerning their cardiac responses towards the four different human emotions. While pet dogs reacted strongest to angry and least to sad expressions, this pattern was reversed for the lab dogs.
\end{abstract}

Interestingly, we found an effect of familiarity only in the lab dogs but not in the pet dogs. Altogether, these results provide physiological support for heterospecific emotional contagion in dogs and highlight the importance of experience in this respect.

Keywords: Heart Rate, Emotion Processing, Domestic Dog

\section{Highlights}

- We demonstrate that dogs' heart rate is influenced by human emotional expressions

- Dogs' living conditions (as pets or lab animals) strongly influences the cardiac response

- The cardiac response of lab, but not pet dogs, is influenced by the familiarity of the human face

\section{INTRODUCTION}

For many socially living species the face serves as a rich source of information [1]. It can provide information about age, gender, attractiveness and the point of attention. Besides this vast amount of references, the face also conveys information about emotions [2]. The ability to perceive and process this information can be of great functional significance, especially in the contexts of threat or reproduction [1,2]. There is evidence that the ability to recognize the emotional expressions of conspecifics has a positive effect on the individual's fitness [3,4]. In addition, it has been shown recently that appropriate response to the emotional status of heterospecific individuals could provide similar functional value [5-7].

ARC Journal of Animal and Veterinary Sciences
The ability to categorize and adaptively react to emotional expressions is accompanied by specific neurophysiological processes, for example the activation of the limbic system via the stimulation of the amygdala [8]. The measurement of such processes in nonhuman animals is of great value not only for the scientific endeavor to understand if and to what extent animals can perceive and react to others' emotions, but also for the society which is increasingly interested to know the cognitive abilities of animals that live in close relation to us, i.e. pets or livestock. A species with particular social and economic value is the dog. A better understanding of its cognitive abilities and emotional states is necessary for ensuring functional human-dog bonds. Unsurprisingly, researchers have investigated not only conspecific (dog-dog) but also heterospecific 
(dog-human) communication and interaction. It has been hypothesized that dogs have developed specialized skills for decoding human communicative cues as a result of a long domestication process $[9,10]$. Indeed, support for this hypothesis comes from several recent studies. For example, dogs are very sensitive towards human communicative signals such as hand gestures and gazing [11]. Dogs are also capable of visually discriminating human faces by exploiting both local and global features [12, 13].

Emotional expressions convey both local and global information. A recent study showed that pet dogs use this information in order to categorize human facial emotions. The dogs not only learned to discriminate half faces - either the lower or the upper semiface - on the basis of the shown emotion (happy vs angry), but demonstrated successful transfer to the other, not previously shown semiface of either familiar or unfamiliar people [14]. The authors concluded that the dogs retrieved their memories about human facial expressions, which they supposedly collected during their individual life and therefore dependent on individual experience. This conclusion was supported by data collected in an eye-tracking study, in which dogs with different rearing and housing histories (pet and lab dogs) showed distinct looking preferences when confronted with human emotional expressions [15]. These differences most likely occurred because pet dogs had ample opportunities to watch the behavior and expressions of their human housemates, while the lab dogs had been kept in small groups in outdoor enclosures and had limited contact to humans. Another recent study revealed that the discrimination of human facial expressions is not simply a perceptual phenomenon but is rather based on a multimodal representation of human emotions [16]. Indirect support for such advanced representations comes from cumulative evidence of a left gaze bias, suggesting a human-like right-hemispheric dominance when processing emotive stimuli $[15,17]$. It is likely that such representations of the emotional expressions of humans are involved in contagious behavior and the ability for a rudimentary form of empathy towards humans and conspecifics [18-24].

Despite converging evidence from dogs' perception of human emotional expressions and their contagious response to them, solid data about physiological correlates of these processes in dogs are still lacking. The prime candidate of these physiological correlates is the autonomous system. It is under influence of the limbic system, of which parts are activated when the organism is confronted with emotional stimuli [25]. Responses of the autonomic nervous system to emotional states can be observed in changes of the heart rate, blood pressure and breathing and they are accompanied by behavioral responses that come along with the emotional state [26-30]. It is presumed that emotions prepare the organism for different actions such as fight and flight via an activation of the sympathicus, or rest and digest via activation of the parasympathicus [31, 32].

From human studies, it is known that responses of the autonomous nervous system differ depending on the experienced emotion. While neutral facial expressions induce very little change in the heart rate, joy and sadness induce some, and anger very strong accelerations [33]. It has been argued that the heart rate deceleration during the perception of sad expression is associated with conservation withdrawal, a basic survival mechanism opposite to fight and flight responses serving to preserve energy [34, 35].

Physiological responses to face stimuli of conspecifics or humans have been investigated in a few animal species. In sheep, for instance, the sight of conspecific faces during social isolation has strong stress- dampening effects, such as reduced activity and protest vocalizations, heart rate, and concentrations of the adrenal hormones cortisol and adrenaline in comparison to pre-isolation levels [36]. A recent study investigated the responses of horses to the presentation of pictures of human emotional facial expressions [7]. The horses' heart rates rose significantly faster when exposed to angry compared with happy faces. Some data about heart rate changes to emotional signals are also available for dogs, but the stimuli were taken from conspecifics and from the tail rather than their faces [37] or were direct measurements of dogs' emotional states but not their reactions towards humans emotions [38, 39].

This study investigated the dog's cardiac responses to human emotional expressions. Beside the effects of different emotions of humans indicated by their facial expressions we were interested in the effect of familiarity with the senders of those signals. In addition to the 
heart rate measurements we examined the looking behavior of these dogs using an eyetracking device [15], therefore the subjects were positioned with their head on a chin rest in front of the face stimuli. With this nearly motionless positioning during stimulus presentation we could control for inadvertent effects of motion and viewing differences. In contrast to most published studies we showed the dogs four instead of only two different facial expressions. This was done to test the occurrence of a graded response as proposed by the arousal- valence model [40]. According to this model we predicted an increase in the strength of the cardiac response from looking at neutral (low valence and low arousal) over sad (intermediate valence and low arousal) and happy (high valence and intermediate arousal) to angry faces (high valence and therefore high arousal). Additionally, we expected differences between familiar and unfamiliar faces with unfamiliar faces eliciting higher heart rates compared to familiar faces, as these faces were unknown and therefore needed subsequent evaluation. To evaluate the influence of individually acquired expertise for human faces and emotions we compared pet dogs that lived for several years in human households and laboratory beagles that lived with conspecifics in enclosures at the University of Veterinary Medicine Vienna. We predicted that the laboratory dogs, in comparison to pet dogs, would show less pronounced differences in their heart rate responses towards different emotional expressions, as these dogs have much less experience with human emotions and consequently lack concrete expectations towards such expressions.

\section{METHODS}

All experimental procedures were discussed and approved by the institutional ethics and animal welfare committee in accordance with GPS guidelines and national legislation at the University of Veterinary Medicine Vienna (approval number: 09/08/97/2012).

\subsection{Subjects}

Seven privately owned dogs (P1-P7) participated in the study. These dogs were of various breeds and age ranged between 1-6 years (mean $=2.43$ years, see Table 1). Additionally, five "laboratory" beagles (L1L5), aged 1-4 years, participated in the study (mean 2.4 years, see Table 1). These dogs were housed in a pack of 13 dogs on the campus of the University of Veterinary Medicine, Vienna.
Table1. List of participating dogs

\begin{tabular}{|c|l|l|l|l|l|}
\hline $\begin{array}{l}\text { Dog } \\
\text { ID }\end{array}$ & Breed & Age $^{(\mathrm{b})}$ & Sex $^{(\mathrm{c})}$ & Status & $\begin{array}{l}\text { Valid } \\
\text { Sessions }\end{array}$ \\
\hline P1 & $\begin{array}{l}\text { Australian } \\
\text { Kelpie }\end{array}$ & 1 & $\mathrm{~m}$ & neutered & 3 \\
\hline P2 & $\begin{array}{l}\text { Australian } \\
\text { Shepherd }\end{array}$ & 3 & $\mathrm{~m}$ & Intact & 3 \\
\hline P3 & $\begin{array}{l}\text { Border } \\
\text { Collie }\end{array}$ & 1 & $\mathrm{~m}$ & Intact & 5 \\
\hline P4 & $\begin{array}{l}\text { German } \\
\text { Shepherd }\end{array}$ & 6 & $\mathrm{f}$ & neutered & 4 \\
\hline P5 & $\begin{array}{l}\text { Hungarian } \\
\text { Viszla }\end{array}$ & 3 & $\mathrm{~m}$ & neutered & 4 \\
\hline P6 & $\begin{array}{l}\text { Mongrel } \\
\text { (Mudi- Schn } \\
\text { auzer-Mix })\end{array}$ & 2 & $\mathrm{f}$ & neutered & 4 \\
\hline P7 & Puli & 1 & $\mathrm{f}$ & intact & 5 \\
\hline L1 & Beagle & 3 & $\mathrm{~m}$ & neutered & 4 \\
\hline L2 & Beagle & 4 & $\mathrm{~m}$ & neutered & 3 \\
\hline L3 & Beagle & 1 & $\mathrm{~m}$ & neutered & 4 \\
\hline L4 & Beagle & 1 & $\mathrm{~m}$ & neutered & 3 \\
\hline L5 & Beagle & 3 & $\mathrm{~m}$ & neutered & 4 \\
\hline
\end{tabular}

Footnotes:
(a) $\mathrm{P}=$ pet $\operatorname{dog}, \mathrm{L}=\operatorname{lab} \operatorname{dog}$
(b) Age in years
(c) $\mathrm{m}=$ male, $\mathrm{f}=$ female

\subsection{Experimental Room and Device}

The experiments were conducted in the eyetracker room of the Clever Dog Lab at the University of Veterinary Medicine, Vienna. This room was divided by a wall, consisting of a projection screen $(200 \times 200 \mathrm{~cm})$ and two doors, into two compartments; a small utility room $(149 \times 356 \mathrm{~cm})$ housing the computer system operating the eye tracker (Eyelink 1000, SR Research, Ontario, Canada) and a video projector (NEC M300XS, NEC Display Solutions, United States), and a larger room $(588 \times 356 \mathrm{~cm})$ with the chin rest device and the eye tracker camera plus LED screen (see Fig. 1; details about the eye- tracking system in Barber et al. 2016).

The dogs were trained to stand on a wooden platform and to put their chin centrally on a customized v- shaped pillow, the chin rest. The chin rest was mounted on a frame of aluminum bars to allow vertical adjustment to the height of the individual dog and placed at a distance of $2 \mathrm{~m}$ from the projection screen (see Fig. 1). The owner of the pet dogs and the experimenter who brought the lab dogs were seated in the back end of the room during the experimental procedure and were instructed to keep interaction with the dog at minimum. 
As a heart rate monitor we used the Polar ${ }^{\circledR}$ RS800CX (Polar Electro GmBH, Oy, Finland), a telemetric and portable measuring system comprised of a wearable chest-belt which includes sensors and transmitters (WearLink and Sender W.I.N.D) that transmit heart beat recordings with a frequency of $1000 \mathrm{~Hz}$ to the trainings computer. To guarantee optimal recordings, dogs' fur and skin and also the chest-belt were moisturized and lubricated with customary ultrasound-gel (Geilo $\mathrm{GmBH}$, Ahaus, Germany). In order to correlate changes in the heart rate with the actual stimuli presentation, we videotaped the experiments with two additional cameras (JVC Everio G GZ MG 750, Yokohama, Japan). One camera was in the front of the chin rest, filming the dogs' head. The second camera was placed behind the chin rest, filming the back of the dog and the projection screen and hence recording the onset of the stimulus presentation. Both cameras were synchronized every session for the exact time with the eye tracking computer and the Polar ${ }^{\circledR}$ monitor.

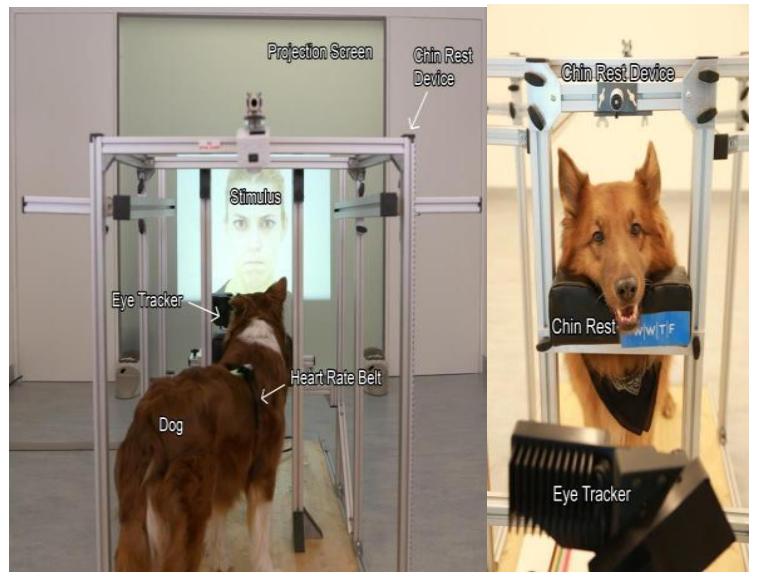

Figure1. The experimental set up. Left: view from the rear of the room with prospect on the chin rest and the projection screen. Right: front view on the chin rest with prospect on the eye tracker

\subsection{Stimuli}

Stimulation of the dog consisted of its owner's and an unfamiliar person's faces expressing four different emotions: neutral, happy, angry and sad. All portrayed humans were female. As the familiar face for the laboratory beagles we used the picture of one of us (DR), who had trained the beagles for the experimental procedure prior to the experiment. Photographs were taken by $\mathrm{AB}$ in a standardized setup using a Canon EOS 6D (Canon Inc., Tokyo, Japan) and studio flash lights (Walimex Pro Studioset VC- 200/200, WALSER GmbHCo. KG, Burgheim, Germany) in front of a white background. The size of the portraits when back-projected onto the projection screen (size projection area: 110 x 80 $\mathrm{cm}$ ) was approximately $400 \times 300 \mathrm{~mm}$.

Stimulus sets were compiled individually for each dog. Unfamiliar owners were assigned semi- randomly to the stimuli sets but owners that differed strongly by their appearance (e.g. red hair and grey hair) and age where not assigned to the stimulus set of the same dog. Each stimulus set consisted of eight different images (familiar: happy, angry, neutral, sad; unfamiliar: angry, happy, neutral, sad) and each image was presented twice during each session.

\subsection{Procedure}

Prior to the experiment, the dogs were trained on the chin-rest device for the experimental procedure. Training lasted between two and six months. We used operant conditioning with positive reinforcement (food rewards) and a clicker as a secondary reinforcement. Dogs were, depending on availability, trained once to twice a week, until they could rest with their snout motionless on the chin rest for at least 30 seconds. For a detailed overview of the training procedure see Barber et al. (2016). In the last five training sessions, the dogs were additionally habituated to the acquisition of the polar belt, including wetting of the fur. Throughout the whole procedure, the dogs were never restrained or forced to perform in the task.

The dogs were tested in five sessions, one per week. At the beginning of a test session the dog could explore the room for $5 \mathrm{~min}$. Then the fur and chest belt were moistened and the chestbelt strapped on the dog with the sensors placed on the cranial part of the thorax, shortly caudal to the elbows. Thereafter, the eye tracking system was calibrated with the procedure explained in Barber et al. (2016). Subsequent to the calibration the dogs obtained a test session.

A test consisted of four stimulus blocks, from which two were comprised of happy and neutral and the other two of angry and sad facial expressions. The order of blocks within a test was randomized. A block consisted of four trials, in which two faces from the familiar and two faces from the unfamiliar person presented. The order of trials within the block was randomized. An attention grabber (small animated video) was presented before every trial. The eye-tracking computer was programmed to present a trial for five seconds or until the dog had been looking at the presented 
stimulus for at least two seconds. After the end of each block, a neutral and pre-trained geometric figure (square or triangle) was presented as a release signal. Following this signal, the dog received a food reward and could leave the chin rest. Depending on the dog's motivation and concentration a pause of one to three minutes was made before the dog was asked to reposition itself in the chin rest. During this pause, the dog could move freely in the room or rest.

\subsection{Data Analysis}

A dog's heart rate was measured as beats per minute (bpm). With the Polar® system we obtained up to three values per second. We defined on- and offset of a trial on the basis of the video recordings and the eye tracking data. To be included in the analyses, data had to fulfil the following criteria: First, the dog had to be attentive during the whole trial, not leaving the chin rest and looking at least once into the face area of the stimulus. Second, to keep a session the dog had to look at least once into faces of both emotional conditions (positive (happy \& neutral) and negative (angry \& sad)). The whole dataset of a dog was excluded if there were less than three valid sessions. Special care was taken to detect and exclude artefacts. Artefacts were defined as extreme values lower than 30 or higher than $250 \mathrm{bpm}$. It has been shown recently that uncorrected Polar ${ }^{\circledR}$ data show excellent accordance to ECG data [41]. Even though there are algorithms to correct dog heart rate data [42], it is still difficult to disentangle other artefacts than extreme values (i.e. interposed extra-systoles, compensated extra-systoles or phase- shifted extra-systoles) without a direct comparison to a ECG measurement [43,44]. Error analysis is therefore dependent on the experience of the researcher and remains nevertheless subjective. For this reason, only extreme values were excluded from the data set and affected data points were deleted instead of interpolated. Following the protocol of Mulder (1992), the summed artefact time of the heart rate data had to be lower than 5\% to keep the session for analysis [45].

Statistical analyses were conducted with SPSS 20 (IBM, New York, USA). Linear mixed effect models (LMM) were used to analyse heart rate. All models included emotion (happy, angry, neutral, sad), familiarity (familiar, unfamiliar) and dog type (pet dog, lab dog) as well as two-way interactions between them, as fixed effects. Where 2-way interactions turned out significant, the dataset was split to explore the nature of the interaction. Dog type (pet or lab dog) and session number nested within dog type were included as random factors in all models. Model reduction was done backwards based on Akaike's information criterion (AIC). Factors were assumed to be significant with a significance level of $\mathrm{p}<0.05$.

\section{Results}

The final dataset included data from 7 pet dogs (4 male and 3 female) with a total of 28 sessions and 19102 data points and 5 lab dogs (all male) with a total of 18 sessions and 18891 data points. This accounts for $76 \%$ of the original data set. Mean heart rate over the whole experiment was $107.66+/-0.24$ bmp (mean +/- SE) in the pet dogs but only $98.89+/$ $0.22 \mathrm{bpm}$ in the lab dogs. This difference was significant (GLMM F1, 12329=237.01, $\mathrm{p}<0.00$ $1)$.

There was neither a significant influence of the familiarity $(\mathrm{F} 1,12329=2.72, \mathrm{p}=0.09)$ nor of the emotion $(\mathrm{F} 1,12329=0.762, \mathrm{p}=0.52)$ of the human faces on the heart rate. However, we found a significant interaction between dog type and emotion $(\mathrm{F} 1,12329=12.94, \mathrm{p}<0.001)$ with the pet dogs showing the highest heart rate for angry and lowest for sad expressions and vice versa for lab dogs (see Fig. 2). As there was a significant difference between the two dog types, both datasets were subsequently examined separately.

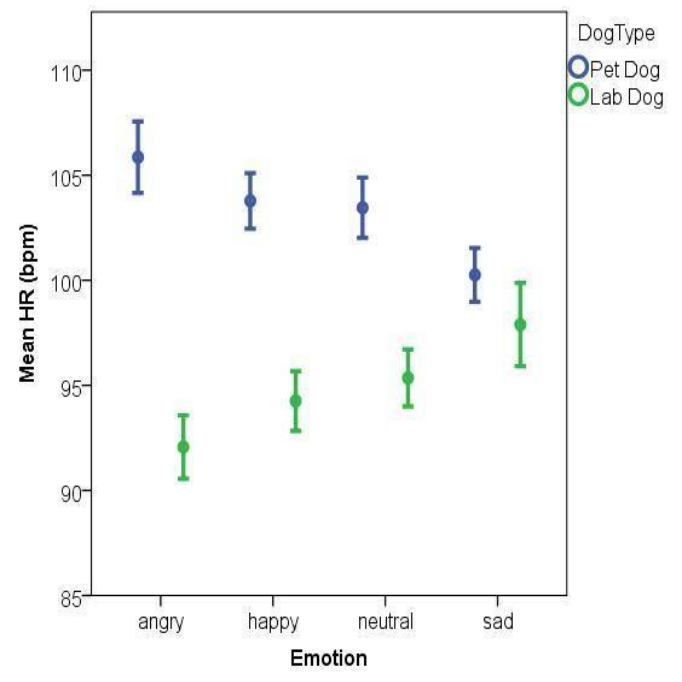

Fig2. Mean heart rate of pet (blue) and lab (green) dog measured during the presentation of faces in four emotions

For the pet dogs, there was a significant influence of the emotion (F3, 7254=6.0, $\mathrm{p}<0.001$, see Fig. 2) but not the familiarity (F1, 
7254=0.004, p=0.9, see Fig. 3) on the heart rate. Additionally, there was a significant interaction between the familiarity and the emotion $(\mathrm{F} 3,7354=7.3, \quad \mathrm{p}<0.001)$ with a significant influence of the familiarity for neutral (familiar>unfamiliar) and happy (familiar< unfamiliar) faces but not for angry and sad faces (happy: $\mathrm{F} 1,1855=4.6, \mathrm{p}=0.03$, neutral: $\mathrm{F} 1,2028=14.9, \mathrm{p}<0.001$, angry: $\mathrm{F} 1,1553=1.2$, $p=0.3$, sad: $F 1,1819=0.45, p=0.5$ ). For the lab dogs, there was a significant influence of the emotion (F3, 5071 =5.9, p $<0.001$, see Fig. 2) and the familiarity $(\mathrm{F} 1,5071=6.8, \mathrm{p}=0.009$, see Fig. 3). Additionally, there was a significant interaction between the familiarity and the emotion $(\mathrm{F} 1,5068=9.3, \quad \mathrm{p}<0.001)$ with a significant influence of the familiarity for all emotional conditions (angry \& sad: familiar> unfamiliar; neutral: familiar<unfamiliar) except happy faces (happy: $F 1,1295=0.04$, $\mathrm{p}=0.8$, neutral: $\mathrm{F} 1,1458=8.6, \mathrm{p}=0.003$, angry: $F 1,1291=20.3, \quad p<0.001, \quad$ sad: $F 1,1027=9.4$, $\mathrm{p}=0.002$ ).

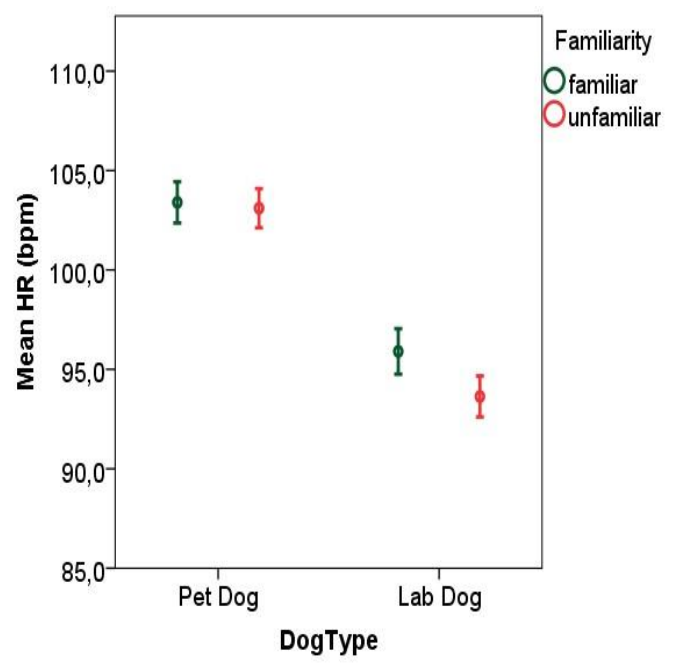

Fig3. Mean heart rate for familiar and unfamiliar faces measured in the group of pet and lab dogs

\section{DISCUSSION}

The aim of this study was to examine the cardiac responses of domestic dogs upon seeing emotional humans in a highly-controlled setting. Rather than confronting free-moving dogs with people, we trained them to stand still with the head put on a chin rest and to watch sequences of pictures showing human faces in four different expressions (happy, neutral, angry and sad). The dogs did indeed show differences in the heart rate when being confronted with human facial expressions, but interestingly in a completely different manner in the two dog types.

\subsection{Influence of the Emotional Expression on the Heart Rate}

We found strong differences of the heart rates between the pet and the lab dogs. The basic difference of the heart rate, with pet dogs having higher heart rates than the lab dogs, can be explained either by breed differences or the lab dogs prior experience with laboratory settings resulting in lower arousal during the laboratory stay compared to the pet dogs. Still, the influence of the emotion of the human faces on the dogs' cardiac response went into the opposite direction in both dog types. The heart rate differences were already visible when the two dog types were confronted with neutral faces, more pronounced with happy faces, but became large with angry faces. In the pet dogs angry expressions elicited the highest heart rate, which is also expected from the literature $[33,34,40]$ and can be explained by an adaptive reaction to a fearful stimulus provoking arousal and therefore acceleration of heart rate due to the activation of the sympathetic system. Interestingly, sad expressions elicited lower heart rate than neutral expressions. This is in conflict to experiments with humans, which have shown that neutral expressions provoke little changes in heart rate responses [33]. There are several explanations for this fact: There have been studies showing that neutral expressions are not perceived as neutral but slightly negative [46]. Neutral expressions are therefore perceived negatively and/or ambivalent and for this reason the heart rate response is higher than expected. Interestingly, sad expressions elicited low heart rates even though it has been shown that dogs react with arousal to crying humans in true-to-life settings [20]. Also it has been shown that dogs' cortisol levels, a measure of distress, are increased upon hearing human infant crying [24]. Even though an audio-visual stimulus like crying is a much stronger stimulus than a static picture, the result of a reduced heart rate response upon seeing pictures of sad expressions could be explained by emotional contagion or forms of empathiclike responses. It has been described that the state of sadness involves conservationwithdrawal or passive coping which is accompanied by inactivity, energy conservation and withdrawal from the environment and therefore characterized by an enhanced parasympathetic activity causing the heart rate to decelerate [34]. Hence, the low heart rate in pet dogs might be a direct result of seeing a sad expression of another individual. 
In the group of the pet dogs the measured heart rate responses towards the emotional expressions are (with the exception of neutral faces) in line with the arousal-valence model (i.e. angry $>$ happy $>$ sad/neutral [40]). The cardiac responses of the lab dogs, in contrast, differed strongly from our expectations. Even though the primary expectation was that the lab dogs' cardiac responses would be due to their poor experiences with human emotions less pronounced between the emotions than in the pet dogs, the experiment revealed a similarly strong effect of the human emotional expressions in both groups of dogs. However, the lab dogs' cardiac responses were reversed in comparison to the pet dogs, i.e. angry faces elicited the lowest heart rate and sad faces the highest heart rate. Most surprising was that angry expressions elicit the lowest heart rate in the lab dogs. The arousal- valence model [40] proposes that anger is an emotion that elicits high arousal and valence. A possible explanation would be that in lab dogs, like in pet dogs, angry facial expressions are perceived as threat and dogs undergo the defense cascade $[47,48]$. According to the defense cascade model an individual undergoes cardiac deceleration when still being in early stages of the defense. Such a phase is initially characterized by a phase of freezing and/or tonic immobility resulting in increased attention and decreased heart rates in the first instance which are subsequently followed by active defense reflexes, accompanied by a further activation of the parasympathetic system and consequently by e.g. an increase of the of heart rate [47-50]. The difference between the heart rates of pet and lab dogs with respect to their response to angry faces might be explained by their experience: Pet dogs have plenty of experience with human facial expressions and might be able to process such expressions faster than lab dogs. Therefore, they are faster in their reaction towards such expressions and react upon such faces with an acceleration of the heart rate - they are already prepared for a fight and flight reaction upon the threat. On the opposite, lab dogs may need first to evaluate the facial expression and are still in the beginning of the fight and flight cascade, resulting in a deceleration of the heart rate - they are in a state of tonic immobility and increased attention. However, further investigation with prolonged stimulus presentation and heart rate measurements of lab and pet dogs are necessary to confirm this hypothesis.
It is also interesting that the heart rates of the lab dogs were higher when presented with neutral and sad expressions than when presented with angry and happy faces. Perhaps due to their lack of experience, lab dogs may perceive these expressions as ambiguous (c.f. [41]). Due to the need for further evaluation of such facial expressions, the dog gets aroused and the heart rate is accelerated. In any case, further investigations are needed to prove if the large differences between pet and lab dogs are a result of their different social environment, which in lab dogs consists mainly of conspecifics but in pet dogs of humans.

\subsection{Influence of the Familiarity of the Heart Rate}

Concerning the familiarity of the face, we collected barely less remarkable findings. While the pet dogs did not show an influence by the familiarity, the lab dogs did. The absence of a familiarity effect has also been previously shown for the processing of facial expressions [14] and could be again explained by the fact that these dogs are much more experienced with human emotions so that familiar and unfamiliar emotional expressions are triggering the same cardiac response. It is, however, less straight forward to explain why in the lab dogs' familiar faces provoked higher heart rate/arousal than unfamiliar faces. As our lab dogs are living in groups of dogs at the campus of the University of Veterinary Medicine Vienna, they have limited contact to humans, which is restricted to feeding and cleaning of the animal keepers, irregular contact with students and veterinarians for practice and contact to humans or other dogs at the fences of their enclosure. The lab dogs therefore had few opportunities to collect associations with different emotions presented by unfamiliar faces. However, in the course of these experiments, the dogs were intensively trained by the experimenter and had the opportunity to collect various experiences with her facial expressions. Additionally, the dogs were able to form multiple associations between the experimenter and the training situations, all of which were based on positive reinforcement. For this reason, the face of the experimenter, which was used as the familiar face, might have been of higher significance for the $\operatorname{dog}$ and consequently provoked more arousal. However, further investigations using both dog types and different levels of familiarity in combination to familiar and 
unfamiliar expressions of con- and heterospecific faces are needed to clarify this result.

\section{CONCLUSION}

This study demonstrates that the physiology of pet and lab dogs is influenced by seeing static pictures of human facial expressions. There are strong differences between the pet and lab dogs suggesting that the social environment of the dog plays an important role respectively in their cardiac response. These results provide the first solid evidence for physiological reactions towards human facial expressions in dogs and should raise the awareness of the importance of emotional cues for non-human animals even in heterospecific contexts. Furthermore, by demonstrating how sensitive dogs are towards our facial expressions, these results have significant implications for human-dog relationships, as they call for special care not only in how we treat them, but also in how we look at them.

\section{ACKNOWLEDGEMENTS}

We are grateful to Simone Grohmann and Denise Ocampo for their help with the experiments, Peter Füreder and Wolfgang Berger for technical help, and Karin Bayer for administrative support. We also thank the dog owners and dogs for participating in this study, and the Clinical Units of Internal MedicineSmall Mammals and Obstetrics, Gynaecology and Andrology for the permission to use their dogs.

\section{FUNDING}

The research presented here was funded by the Vienna Science and Technology Funds (WWTF), No. CS11-005; http://www.wwtf.at/) to Ludwig Huber

\section{REFERENCES}

[1] C. Darwin, The Expression of the Emotions in Man and Animals, 1965. doi:10.1097/00000441- 195610000-00024.

[2] P. Ekman, W. V Friesen, Unmasking the face: A guide to recognizing emotions from facial clues, Malor Books, Cambridge, 1975. http:// psycnet.apa.org/psycinfo/1975-31746-000.

[3] L.A. Parr, The Discrimination of Faces and Their Emotional Content by Chimpanzees (Pan troglodytes), in: Ann. N. Y. Acad. Sci., 2003: pp. 56-78. doi:10.1196/annals.1280.005.

[4] V. Dufour, O. Pascalis, O. Petit, Face processing limitation to own species in primates: A comparative study in brown capuchins, Tonkean macaques and humans,
Behav. Processes. 73 (2006) 107-113. doi:10. 1016/j.beproc.2006.04.006.

[5] R.D. Magrath, T.M. Haff, P.M. Fallow, A.N. Radford, Eavesdropping on heterospecific alarm calls: from mechanisms to consequences, Biol. Rev. 90 (2015) 560-586. doi:10.1111/brv.12122.

[6] T.M. Haff, R.D. Magrath, Learning to listen? Nestling response to heterospecific alarm calls, Anim. Behav. 84 (2012) 1401-1410. doi:10.1016/j.anbehav.2012.09.005.

[7] A.V. Smith, L. Proops, K. Grounds, J. Wathan, K. McComb, Functionally relevant responses to human facial expressions of emotion in the domestic horse ( Equus caballus ), Biol. Lett. 12 (2016) 20150907. doi:10.1098/ rsbl. 2015. 0907.

[8] P. Ekman, Basic Emotions, in: Handb. Cogn. Emot., 1999: pp. 45-60.

[9] B. Hare, M. Tomasello, Human-like social skills in dogs?, Trends Cogn. Sci. 9 (2005) 439-444. doi:10.1016/j.tics.2005.07.003.

[10] E. Téglás, A. Gergely, K. Kupán, Á. Miklósi, J. Topál, Dogs' gaze following is tuned to human communicative signals, Curr. Biol. 22 (2012)

209-212. doi:10.1016/j.cub.2011.12.018.

[11] J. Kaminski, L. Schulz, M. Tomasello, How dogs know when communication is intended for them, Dev. Sci. 15 (2012) 222-232. doi:10.1111/j.1467-7687.2011.01120.x.

[12] L. Huber, A. Racca, B. Scaf, Z. Virányi, F. Range, Discrimination of familiar human faces in dogs (Canis familiaris), Learn. Motiv. 44 (2013) 258-269. doi:10.1016/j.lmot.2013.04.005.

[13] E. Pitteri, P. Mongillo, P. Carnier, L. Marinelli, L. Huber, Part-based and configural processing of owner's face in dogs., PLoS One. 9 (2014) e108176. doi:10.1371/ journal. pone.0108176.

[14] C.A. Müller, K. Schmitt, A.L.A. Barber, L. Huber, Dogs can discriminate emotional expressions of human faces, Curr. Biol. 25 (2015) 601-605. doi:10.1016/j.cub.2014.12. 055 .

[15] A.L.A. Barber, D. Randi, C.A. Müller, L. Huber, The Processing of Human Emotional Faces by Pet and Lab Dogs: Evidence for Lateralization and Experience Effects, PLoS One. 11 (2016) e0152393. doi:10.1371/journal .pone.0152393.

[16] N. Albuquerque, K. Guo, A. Wilkinson, C. Savalli, E. Otta, D. Mills, Dogs recognize dog and human emotions, Biol. Lett. 12 (2016) 20150883. doi:10.1098/rsbl.2015.0883.

[17] A. Racca, K. Guo, K. Meints, D.S. Mills, Reading faces: Differential lateral gaze bias in processing canine and human facial expressions in dogs and 4-year-old children, PLoS One. 7 (2012) 1-10. doi:10.1371/journal .pone.0036076. 
[18] A. Huber, A.L.A. Barber, T. Faragó, C.A. Müller, L. Huber, Investigating emotional contagion in dogs (Canis familiaris) to positively and negatively emotional sounds of humans and conspecifics, Anim. Cogntiion. submitted (2017).

[19] K. Silva, L. de Sousa, "Canis empathicus"? A proposal on dogs' capacity to empathize with humans., Biol. Lett. 7 (2011) 489-492. doi:10. 1098/rsbl.2011.0083.

[20] D. Custance, J. Mayer, Empathic-like responding by domestic dogs (Canis familiaris) to distress in humans: an exploratory study., Anim. Cogn. (2012). doi:10.1007/s10071-0120510-1.

[21] Z. Sümegi, K. Oláh, J. Topál, Emotional contagion in dogs as measured by change in cognitive task performance, Appl. Anim. Behav. Sci. 160 (2014) 106-115. doi:10.1016/ j.applanim.2014.09.001.

[22] E. Palagi, V. Nicotra, G. Cordoni, Rapid mimicry and emotional contagion in domestic dogs., R. Soc. Open Sci. 2 (2015) 150505. doi:10.1098/rsos.150505.

[23] M. Quervel-Chaumette, V. Faerber, T. Faragó, S. Marshall-Pescini, F. Range, Investigating Empathy- Like Responding to Conspecifics' Distress in Pet Dogs., PLoS One. 11 (2016) e0152920. doi:10.1371/journal.pone.0152920.

[24] M.H. Yong, T. Ruffman, Emotional contagion: Dogs and humans show a similar physiological response to human infant crying, Behav. Processes. 108 (2014) 155-165. doi:10.1016/ j. beproc.2014.10.006.

[25] A. Boissy, G. Manteuffel, M.B. Jensen, R.O. Moe, B. Spruijt, L.J. Keeling, C. Winckler, B. Forkman, I. Dimitrov, J. Langbein, M. Bakken, I. Veissier, A. Aubert, Assessment of positive emotions in animals to improve their welfare, Physiol. Behav. 92 (2007) 375-397. doi: 10. 1016/j.physbeh.2007.02.003.

[26] M. V Kujala, Canine emotions as seen through human social cognition, Anim. Sentience. 13 (2017) 1- 34. http://animalstudiesrepository. org/animsent/guidelines.html.

[27] N. Ogata, T. Kikusui, Y. Takeuchi, Y. Mori, Objective measurement of fear-associated learning in dogs, J. Vet. Behav. Clin. Appl. Res. 1 (2006) 55-61. doi:10.1016/ j.jveb. 2006. 06.002 .

[28] T. King, P.H. Hemsworth, G.J. Coleman, Fear of novel and startling stimuli in domestic dogs, Appl. Anim. Behav. Sci. 82 (2003) 45-64. doi:10.1016/S0168-1591(03)00040-6.

[29] E. Hydbring-Sandberg, L.W. von Walter, K. Höglund, K. Svartberg, L. Swenson, B. Forkman, Physiological reactions to fear provocation in dogs, J. Endocrinol. 180 (2004) 439-448. doi:10.1677/joe.0.1800439.
[30] D.J. Anderson, R. Adolphs, A framework for studying emotions across species, Cell. 157 (2014) 187- 200. doi:10.1016/ j.cell. 2014. 03.003 .

[31] J.B. Reece, L.A. Urry, M.L. Cain, S.A. Wasserman, P. V. Minorsky, R.B. Jackson, Campbell Biology, 2010. doi:10.1007/s13398014-0173-7.2.

[32] G. Pocock, C. Richards, Human Physiology the Basis of Medicine, Ulster Med. J. 77 (2008) 216. doi:10.1136/bjsm.2006.026120.

[33] R. Sinha, W.R. Lovallo, O. a Parsons, Cardiovascular differentiation of emotions., Psychosom. Med. 54 (1992) 422-435. doi:10.1097/00006842-199207000-00005.

[34] S.D. Kreibig, F.H. Wilhelm, W.T. Roth, J.J. Gross, Cardiovascular, electrodermal, and respiratory response patterns to fear- and sadness-inducing films, Psychophysiology. 44 (2007) 787-806. doi:10.1111/j.1469-8986. 2007.00550.x

[35] G.L. Engel, A.H. Schmale, Conservationwithdrawal: a primary regulatory process for organismic homeostasis., Ciba Found. Symp. 8 (1972) 57-75. http://www.ncbi.nlm.nih.gov/ pubmed/4144967 (accessed October 25, 2016).

[36] A.P. da Costa, A.E. Leigh, M.-S. Man, K.M. Kendrick, Face pictures reduce behavioural, autonomic, endocrine and neural indices of stress and fear in sheep., Proc. Biol. Sci. 271 (2004) 2077-2084. doi:10.1098/ rspb. 2004. 2831.

[37] M. Siniscalchi, R. Lusito, G. Vallortigara, A. Quaranta, Seeing left- or right-asymmetric tail wagging produces different emotional responses in dogs, Curr. Biol. 23 (2013) 22792282. doi:10.1016/j.cub.2013.09.027.

[38] M. Katayama, T. Kubo, K. Mogi, K. Ikeda, M. Nagasawa, T. Kikusui, Heart rate variability predicts the emotional state in dogs, Behav. Processes. 128 (2016) 108-112. doi:10.1016/ j. beproc.2016.04.015.

[39] F. Kuhne, J.C. Hößler, R. Struwe, Emotions in dogs being petted by a familiar or unfamiliar person: Validating behavioural indicators of emotional states using heart rate variability, Appl. Anim. Behav. Sci. 161 (2014) 113-120. doi:10.1016/j.applanim.2014.09.020.

[40] J.A. Russell, A Circumplex Model of Affect, J. Pers. Soc. Psychol. 39 (1980) 1161-1178. doi:10.1037/h0077714.

[41] A. Essner, R. Sjöström, E. Ahlgren, B. Lindmark, Validity and reliability of Polar ${ }^{\circledR}$ RS800CX heart rate monitor, measuring heart rate in dogs during standing position and at trot on a treadmill, Physiol. Behav. 114-115 (2013) 1-5. doi:10.1016/j.physbeh.2013.03.002. 
[42] I. Schöberl, K. Kortekaas, F.F. Schöberl, K. Kotrschal, Algorithm-supported visual error correction (AVEC) of heart rate measurements in dogs, Canis lupus familiaris, Behav. Res. Methods. 47 (2015) 1356-1364. doi:10.3758/ s13428-014-0546-z.

[43] R.M. Marchant-Forde, D.J. Marlin, J.N. Marchant-Forde, Validation of a cardiac monitor for measuring heart rate variability in adult female pigs: Accuracy, artefacts and editing, Physiol. Behav. 80 (2004) 449-458. doi:10.1016/j.physbeh.2003.09.007.

[44] E. von Borell, J. Langbein, G. Després, S. Hansen, C. Leterrier, J. Marchant-Forde, R. Marchant-Forde, M. Minero, E. Mohr, A. Prunier, D. Valance, I. Veissier, Heart rate variability as a measure of autonomic regulation of cardiac activity for assessing stress and welfare in farm animals - A review, Physiol. Behav. 92 (2007) 293-316. doi:10.10 16/j.physbeh.2007.01.007.

[45] L.J.M. Mulder, Measurement and analysis methods of heart rate and respiration for use in applied environments, Biol. Psychol. 34 (1992) 205-236. doi:10.1016/0301-0511(92)90016-N.

[46] E. Lee, J.I. Kang, I.H. Park, J.-J. Kim, S.K. An, Is a neutral face really evaluated as being emotionally neutral?, Psychiatry Res. 157 (2008) 77-85. doi:10.1016/j.psychres. 2007. 02.005 .

[47] M.M. Bradley, M. Codispoti, B.N. Cuthbert, P.J. Lang, Emotion and motivation I: defensive and appetitive reactions in picture processing, Emotion. 1 (2001) 276-298. doi:10.1037/15283542.1.3.276.

[48] K. Kozlowska, P. Walker, L. McLean, P. Carrive, Fear and the Defense Cascade, Harv. Rev. Psychiatry. 23 (2015) 1. doi:10.1097/ HRP.0000000000000065.

[49] P.J. Lang, M.M. Bradley, B.N. Cuthbert, Emotion, attention, and the startle reflex., Psychol. Rev. 97 (1990) 377-395. doi:10.1037/ 0033-295X.97.3.377.

[50] P.J. Lang, The Emotion Probe, Am. Psychol. Assoc. 50 (1995) 372-385. doi:10.1037/0003066X.50.5.372.

Citation: Anjuli L. A. Barber*, Ester M. Müller, Dania Randi, Corsin A. Müller, Ludwig Huber. Heart Rate Changes in Pet and Lab Dogs as Response to Human Facial Expressions. ARC Journal of Animal and Veterinary Sciences. 2017; 3(2):46-55. doi: dx.doi.org/ 10.20431/2455-2518.0302005.

Copyright: (C) 2017 Authors. This is an open-access article distributed under the terms of the Creative Commons Attribution License, which permits unrestricted use, distribution, and reproduction in any medium, provided the original author and source are credited. 